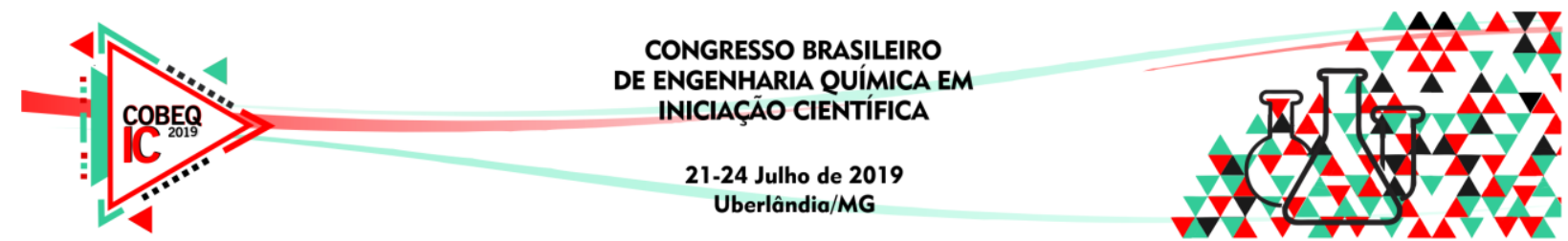

\title{
ANÁLISE DE OPERABILIDADE APLICADA A UM SISTEMA DE QUATRO TANQUES COM FALHAS EM ATUADORES
}

\author{
P. H. V. REZENDE ${ }^{1}$, M. G. B. MELO ${ }^{1}$ e T. V. $\operatorname{COSTA}^{1}$ \\ ${ }^{1}$ Universidade Federal de Itajubá, Instituto de Recursos Naturais \\ E-mail para contato: thiagocosta@unifei.edu.br
}

\begin{abstract}
RESUMO - Processos químicos industriais são altamente dependentes da estrutura de controle para garantia de sua segurança e rentabilidade. Logo, a ocorrência de uma falha no sistema de controle da planta pode levar a prejuízos materiais e pessoais de grandes proporções. Este trabalho tem por objetivo a aplicação de ferramentas baseadas no estudo de operabilidade de processos em falha, com a eventual perda de um de seus atuadores. Com isso, pretende-se validar sua aplicação na determinação sistemática de restrições para as variáveis controladas, necessárias à implementação de estratégias de controle tolerante, para o realinhamento dos objetivos de controle perante a situação de falha. Foram analisados casos de travamento das bombas de alimentação de um sistema de tanques, em que se obteve, por meio da análise de operabilidade, as faixas em que as variáveis controladas poderiam ser mantidas sem que o processo se torne inoperável, quando submetido aos valores estipulados de perturbações. Os resultados indicam a factibilidade da aplicação de tais ferramentas para a determinação das máximas restrições aplicáveis às variáveis controladas, bem como sua possível contribuição ao desenvolvimento de estratégias de controle tolerante.
\end{abstract}

\section{INTRODUÇÃO}

A ocorrência de falhas na instrumentação de processos químicos pode levar ao comprometimento da produção, afetando também a segurança e a rentabilidade do processo, em vista da interdependência entre os elementos da malha para o controle da planta. A vulnerabilidade do sistema de controle às falhas impulsiona estudos relacionados a este tema, sobretudo no desenvolvimento de ferramentas que mantenham a operabilidade do processo diante de um cenário de falha, o que define a viabilidade de se implementar estratégias que refreiam a sua propagação, permitindo, dessa forma, que tais falhas possam ser acomodadas. Um caso mais específico trata da perda de um dos atuadores do processo, o que impede o controle das variáveis de interesse em seus pontos ótimos de operação. Para um sistema sem redundâncias, soluções estudadas nesse contexto apontam para o controle das variáveis de interesse em faixas de valores próximos aos objetivos de controle originais, de forma a minimizar o desvio associado à falha e garantir a estabilidade do processo (Benosman, 2010). Tal estratégia é aplicada levando-se em conta as limitações físicas dos atuadores remanescentes, que passam a atuar de forma compensatória. 


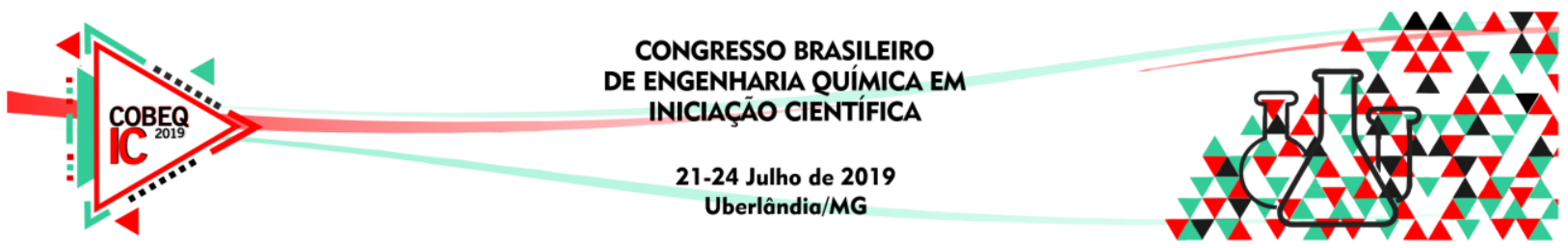

As considerações feitas sobre as restrições associadas ao processo, devido às limitações dos atuadores da malha, ou então, determinadas em favor da sua eficiência e segurança, são outro ponto-chave para a aplicação da estratégia de controle tolerante, visto que a falha pode levar à violação dessas restrições. Destaca-se, aqui, o estudo realizado por Lima e Georgakis (2007), que analisa a operabilidade de sistemas não quadrados, identificando as mínimas restrições aplicáveis às variáveis controladas, levando em conta as possíveis perturbações inerentes ao processo. Tais restrições são de particular interesse para a implementação de estratégias de controle avançado, a exemplo do controle preditivo baseado em modelo (model predictive control, MPC) (Mayne et al., 2000).

O objetivo deste trabalho consiste, portanto, na aplicação da análise de operabilidade proposta por Lima e Georgakis (2007) no contexto de falhas, para determinação sistemática das restrições associadas às variáveis controladas de um processo com falha em um dos atuadores da malha de controle. Tal aplicação contribuirá, em trabalhos futuros, para a implementação da estratégia de controle tolerante nos casos estudados. Nesse contexto, propõe-se determinar a capacidade de operação do sistema com falhas em seus atuadores, permitindo compreender qual será o seu comportamento quando submetido a perturbações.

\section{FUNDAMENTAÇÃO TEÓRICA}

A análise de operabilidade de um sistema com $n_{u}$ entradas, $n_{y}$ saídas e $n_{d}$ perturbações é aplicada em Lima e Georgakis (2007) através da relação entre os intervalos considerados para o conjunto de valores de saída desejados (DOS, Desired Output Set) e o conjunto de valores de saída possíveis do sistema (AOS, Achievable Output Set). Da interseção de tais intervalos, obtém-se um outro conjunto, definido como intervalo do conjunto de valores de saída possíveis (AOIS, Achievable Output Interval Set), de mesma proporção do DOS, limitado pelas restrições de valor máximo e mínimo do AOS. Para um sistema operável, é de interesse determinar a razão de hipervolume (HVR), calculada de acordo com a Equação 1:

$$
H V R=\frac{\mu(D O S)}{\mu(A O I S)}
$$

Em que $\mu$ representa a função que calcula o tamanho dos intervalos, a exemplo da área, no caso de intervalos de duas dimensões. O conjunto de valores de saída desejados (DOS) representa os valores desejados para as variáveis controladas do processo, cujos limites são baseados em parâmetros operacionais e econômicos, determinado pela Equação 2. O conjunto de valores de saída possíveis (AOS) é determinado por um conjunto de valores de entrada disponíveis (AIS, Available Input Set), que corresponde ao conjunto de valores em que as variáveis de entrada possam ser mantidas, tendo como limite as restrições operacionais geralmente associadas aos limites físicos de operação dos atuadores; o conjunto AOS depende, também, de um conjunto de valores de possíveis perturbações aos quais o sistema pode ser submetido (EDS, Expected Disturbance Set) (Lima e Georgakis, 2007). Os intervalos destes conjuntos são apresentados nas Equações 3, 4 e 5:

$$
D O S=\left\{Y \mid y_{i}^{\min } \leq y_{i} \leq y_{i}^{\max } ; 1 \leq i \leq n_{y}\right\}
$$




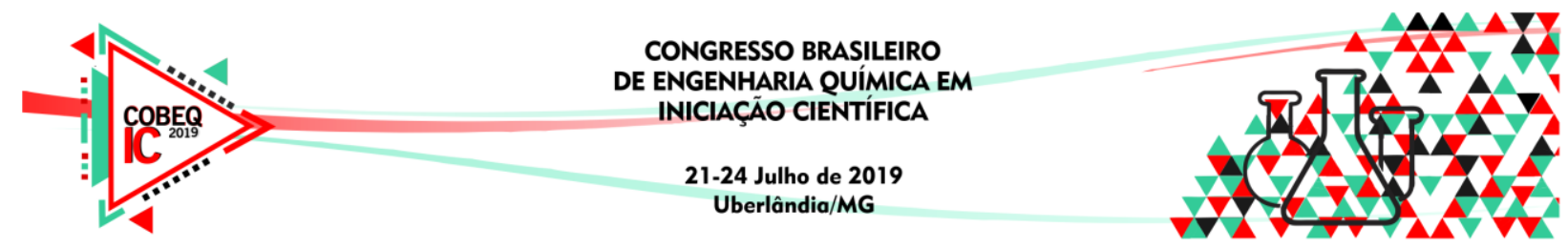

$$
\begin{gathered}
A O S=\left\{Y \mid Y=\underline{K} \cdot U+\underline{K}_{d} \cdot D ; U \in A I S, D \in E D S\right\} \\
A I S=\left\{U \mid u_{i}^{\text {min }} \leq u_{i} \leq u_{i}^{\text {max }} ; 1 \leq i \leq n_{u}\right\} \\
E D S=\left\{D \mid d_{i}^{\text {min }} \leq d_{i} \leq d_{i}^{\max } ; 1 \leq i \leq n_{d}\right\}
\end{gathered}
$$

Com $Y \in \mathbb{R}^{n_{y}}, U \in \mathbb{R}^{n_{u}}$ e $D \in \mathbb{R}^{n_{d}} ;$ sendo $\underline{K}$ a matriz de ganhos do sistema e $\underline{K}_{d}$ a matriz de ganhos das perturbações.

O valor do HVR, maior que um para processos operáveis, pode ser interpretado como a proporção a que as variáveis de saída podem ser restringidas, em relação às restrições prédeterminadas do DOS, sem que isso cause inviabilidades no processo. Para o caso em que HVR $<1$, tem-se que o processo é inoperável e, dessa forma, deve-se reavaliar as restrições pré-determinadas às variáveis de saída para a garantia de controlabilidade do processo (Lima e Georgakis, 2007).

\section{ESTUDO DE CASO}

Foi tomado como objeto de análise um sistema de quatro tanques (Johansson, 2000), recorrente na literatura de controle de processos em virtude de sua versatilidade, a exemplo do estudo apresentado em Berner et al. (2018). Fez-se a análise de operabilidade desse sistema, de acordo com o modelo dinâmico apresentado em Vadigepalli, Gatzke e Doyle III (2001), em que se deseja controlar os níveis de dois dos tanques por meio do ajuste da velocidade de duas bombas que alimentam o sistema. Trata-se de um processo quadrado, isto é, com o mesmo número de entradas e saídas $(2 \times 2)$. O cenário sob o qual é feita a análise é o de uma possível falha em um dos atuadores do sistema, em que a potência de uma das bombas permanece bloqueada no estado estacionário, tornando-o um sistema não quadrado. A análise de operabilidade subsequente determina se o sistema ainda é operável nesse caso, evidenciando os limites possíveis de serem implementados nas variáveis de saída para que o sistema opere o mais próximo de sua condição nominal, obtidos por representação dos conjuntos de valores no $\mathrm{R}^{2}$.

O modelo dinâmico do sistma é representado por um conjunto de equações não-lineares, composto pelas Equações de 6 a 9 .

$$
\begin{gathered}
\frac{d h_{1}}{d t}=\frac{a \sqrt{2 g}\left(\sqrt{h_{3}}-\sqrt{h_{1}}\right)+\gamma_{1} \cdot k_{1} \cdot v_{1}-K d_{1} \cdot d_{1}}{A} \\
\frac{d h_{2}}{d t}=\frac{a \sqrt{2 g}\left(\sqrt{h_{4}}-\sqrt{h_{2}}\right)+\gamma_{2} \cdot k_{2} \cdot v_{2}-K d_{2} \cdot d_{2}}{A} \\
\frac{d h_{3}}{d t}=\frac{\left(1-\gamma_{2}\right) \cdot k_{2} \cdot v_{2}-a \sqrt{2 g \cdot h_{3}}}{A}
\end{gathered}
$$




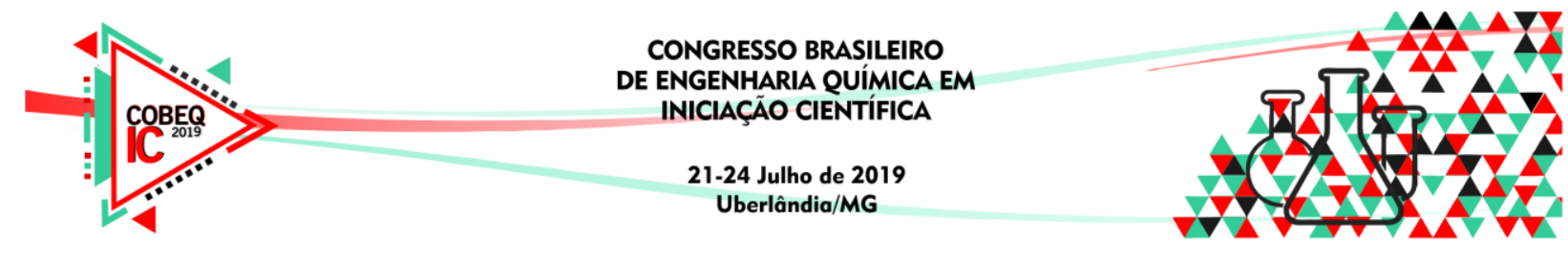

$$
\frac{d h_{4}}{d t}=\frac{\left(1-\gamma_{1}\right) \cdot k_{1} \cdot v_{1}-a \sqrt{2 g \cdot h_{4}}}{A}
$$

No modelo, $h_{i}$ é o valor do nível do tanque $i$; $a$ é a área da seção transversal da tubulação de saída do tanque; $A$ é a área de seção transversal dos tanques; $v_{j}$ é a velocidade nominal do fluido bombeado pela bomba $j$ com o ganho correspondente $k_{j} ; \lambda_{j}$ é parte da vazão que segue para os tanques superiores saindo da bomba $j ; g$, a gravidade. Foram consideradas perturbações na vazão dos tanques inferiores, representadas pelas variáveis $d_{1}$ e $d_{2}$, com os ganhos repectivos $K d_{1}$ e $K d_{2}$. Os valores dos parâmetros e condições de operação do sistema foram os mesmos utilizados em Vadigepalli, Gatzke e Doyle III (2001).

A matriz de ganhos do sistema é representado pela Equação 10:

$$
\left[\begin{array}{l}
h_{1}{ }^{\prime} \\
h_{2}{ }^{\prime}
\end{array}\right]=\left[\begin{array}{ll}
0,78 & 0,50 \\
0,54 & 1,18
\end{array}\right]\left[\begin{array}{l}
v_{1}{ }^{\prime} \\
v_{2}{ }^{\prime}
\end{array}\right]+\left[\begin{array}{l}
-0,05 \\
-0,06
\end{array}\right]\left[\begin{array}{l}
d_{1}{ }^{\prime} \\
d_{2}{ }^{\prime}
\end{array}\right]
$$

Dois cenários de falha distintos são considerados, resultando nos valores de potência da bomba $1 \mathrm{e}$, posteriormente, da bomba 2 travados no estado estacionário $\left(v_{1}{ }^{\prime}=0 ; v_{2}{ }^{\prime}=0\right)$. Considera-se, então, que é desejável manter os níveis dos tanques 1 e 2 em uma faixa de intervalos de $10 \mathrm{~cm}$ em relação ao estado nominal, o que representa restrições operacionais associadas aos valores de saída do sistema. $\mathrm{O}$ atuador remanescente no sistema tem um limite determinado pela porcentagem de potência, de 0 a $100 \%$ (-50\% a 50\%, considerando o seu deslocamento em relação ao estado estacionário) e as perturbações do sistema foram estipuladas num intervalo de -100 a 100. Com base nessas considerações, define-se os conjuntos de valores de entrada disponíveis (AIS), valores de saída desejados (DOS) e valores de perturbações esperados (EDS), apresentados nas Equações de 11 a 13:

$$
\begin{gathered}
D O S=\left\{H \mid-10 \leq h_{1}{ }^{\prime} \leq 10 ;-10 \leq h_{2}{ }^{\prime} \leq 10\right\} \\
A I S=\left\{V \mid-50 \leq v_{1}{ }^{\prime} \leq 50 ;-50 \leq v_{2}{ }^{\prime} \leq 50\right\} \\
E D S=\left\{D \mid-100 \leq d_{1}{ }^{\prime} \leq 100 ;-100 \leq d_{2}{ }^{\prime} \leq 100\right\}
\end{gathered}
$$

Com $H=\left[h_{1}{ }^{\prime}, h_{2}{ }^{\prime}\right]^{T}, V=\left[v_{1}{ }^{\prime}, v_{2}{ }^{\prime}\right]^{T}$ e $D=\left[d_{1}{ }^{\prime}, d_{2}{ }^{\prime}\right]^{T}$.

Após a ocorrência da falha, o sistema se torna não-quadrado, com um menor número de entradas em relação às saídas, sendo possível representar a sobreposição de tais conjuntos num plano de coordenadas cartesianas, como mostram as Figuras de 1 e 2, para os casos de falha nos atuadores das bombas 1 e 2, respectivamente. Nas Figuras 1 (i) e 2 (i), são apresentados os conjuntos AOS, obtidos através da Equação 3, com base nos valores extremos esperados para o par de perturbações do sistema (linhas a, b, c e d), juntamente com o intervalo DOS. A união dos conjuntos AOS calculados em função de todos os valores das perturbações dentro das faixas previamente estipuladas formam o planos representados nas Figuras 1 (ii) e 2 (ii). A interseção entre os planos de DOS e AOS formam o intervalo AOIS. 


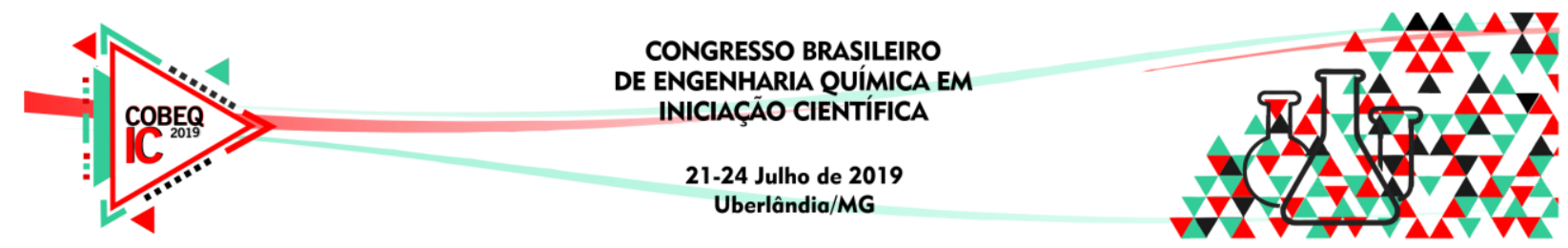

Figura 1 - Representação dos conjuntos DOS e AOS e do intervalo AOIS para o cenário de falha na bomba 1.

(i)

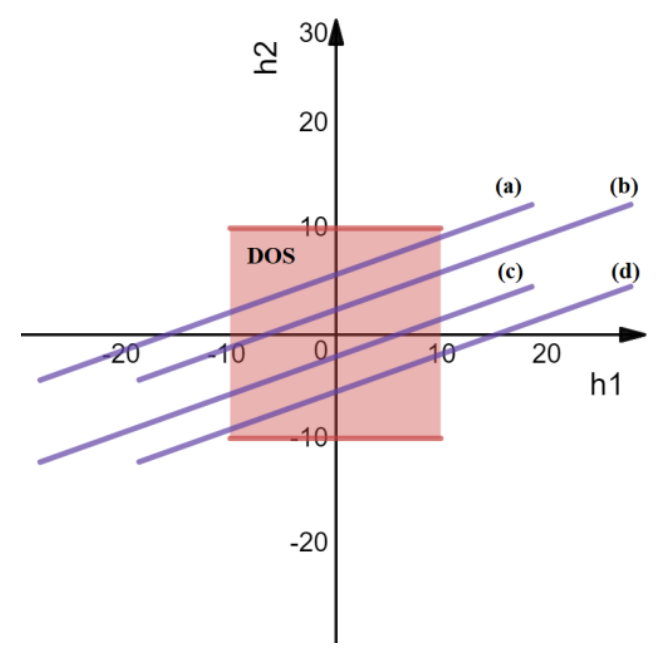

(ii)

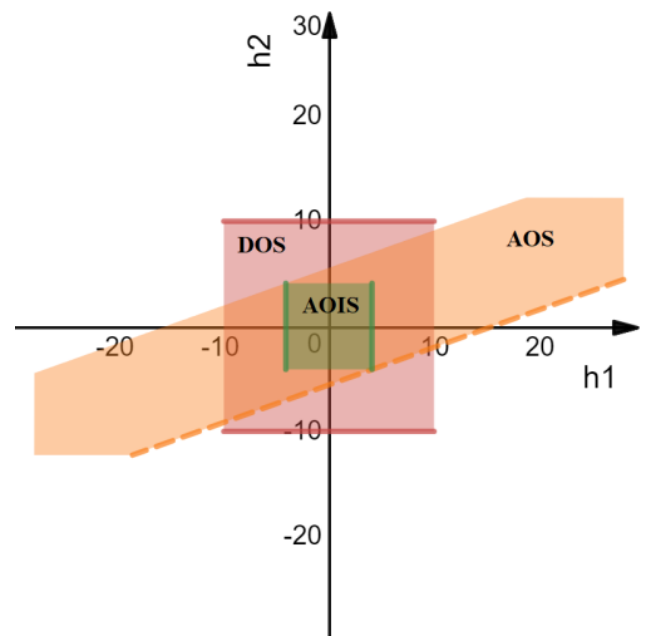

(a) $\operatorname{AOS}\left(\mathrm{d}_{1}=100, \mathrm{~d}_{2}=-100\right)$; (b) AOS $\left(\mathrm{d}_{1}=-100, \mathrm{~d}_{2}=-100\right)$; (c) AOS $\left(\mathrm{d}_{1}=100, \mathrm{~d}_{2}=100\right)$; (d) AOS $\left(\mathrm{d}_{1}=-100\right.$, $\left.\mathrm{d}_{2}=100\right)$.

Figura 2 - Representação dos conjuntos DOS e AOS e do intervalo AOIS para o cenário de falha na bomba 2.

(i)

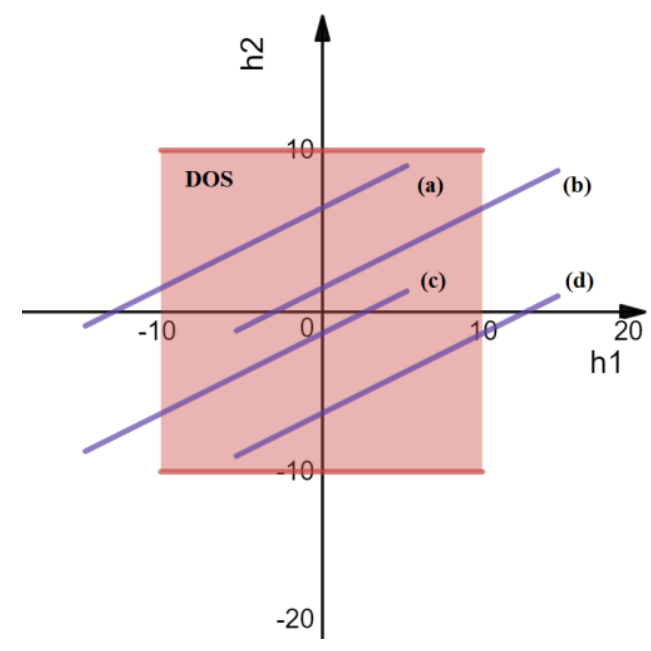

(ii)

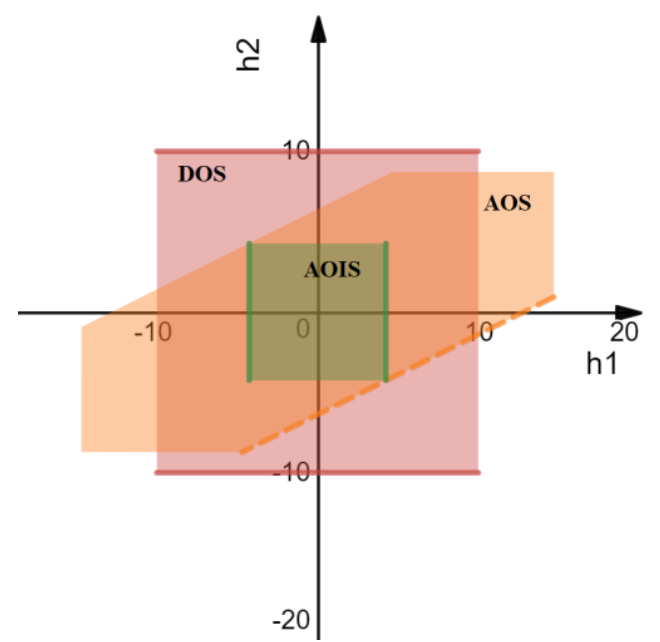

(a) AOS $\left(d_{1}=100, d_{2}=-100\right)$; (b) AOS $\left(d_{1}=-100, d_{2}=-100\right)$; (c) AOS $\left(d_{1}=100, d_{2}=100\right)$; (d) AOS $\left(d_{1}=-100\right.$, $\left.\mathrm{d}_{2}=100\right)$.

Tem-se, então, que o sistema é operável mesmo após a ocorrência da falha em um de seus atuadores, com base nas representações das Figuras 1 (ii) e 2 (ii), pois o intervalo de valores de saída possíveis (AOIS) está contido na faixa de valores desejáveis (DOS), em que os níveis dos tanques 1 e 2 deveriam ser matidos. Analisando os valores que definem o limite 


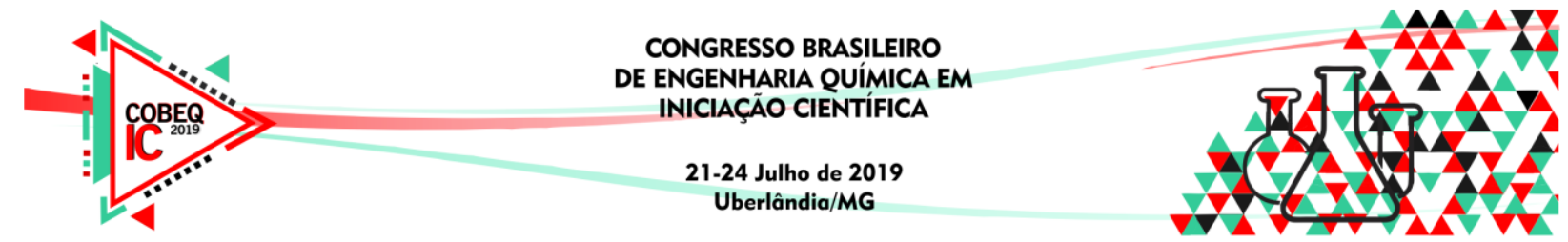

do AOIS em cada caso, temos as restrições máximas aplicáveis às saídas $h_{1}$ ' e $h_{2}$ ', definidas por: $-4,10 \leq h_{1}{ }^{\prime}, h_{2}{ }^{\prime} \leq 4,10$ no caso de falha em $v_{1}{ }^{\prime}$, e-4,26 $\leq h_{1}{ }^{\prime}, h_{2}{ }^{\prime} \leq 4,26$, no caso de falha em $v_{2}$ '.

Calcula-se a razão HVR entre os conjuntos de DOS e AOIS para cada caso, resultando em 5,94 para o caso (i) e 5,51 para o caso (ii), o que o define, novamente, como um processo controlável para tais cenários de falha, uma vez que $H V R \geq 1$, indicando que as restrições aplicadas a $h_{1}$ ' e $h_{2}$ ' podem ser ainda menores. Tal informação é de grande valia para a implementação de uma estratégia de controle tolerante que associe tais limites à lógica de reconfiguração do controlador para acomodação da falha.

\section{CONCLUSÃO}

Este trabalho teve como objetivo analisar a controlabilidade de um sistema de quatro tanques quando submetido a uma falha em um de seus atuadores. As ferramentas de análise de operabilidade desenvolvidas por Lima e Georgakis (2007) se mostram aplicáveis para o estudo de caso considerado, uma vez que foi possível obter, para os cenários de falha considerados, uma verificação da operabilidade do sistema e de seu comportamento diante das perturbações esperadas. Foi possível, também, definir com base na análise da sobreposição dos conjuntos no $\mathrm{R}^{2}$, as faixas de operação para as variáveis de nível a serem controladas, informação, esta, de grande aplicabilidade para a implementação de estratégias de controle mais avançadas, que utilizam de tais restrições na solução do problema de otimização associado à dinâmica do sistema em falha, como no caso do controle tolerante a falhas, garantindo que o sistema opere de forma minimamente satisfatória.

\section{REFERÊNCIAS}

BENOSMAN, M. A survey of some recent results on nonlinear fault tolerant control. Mathematical Problems in Engineering, 2009, v. 2010, 25p.

BERNER, J.; SOLTESZ, K.; HAGGLUND, T.; ASTROM, K. J. An experimental comparison of PID autotuners. Control Engineering Practice, v. 73, p. 124-133, 2018.

JOHANSSON, K. H. The quadruple-tank process: A multivariable laboratory process with an adjustable zero. IEEE Transactions on Control Systems Technology, v. 8, n. 3, p. 456-465, 2000 .

LIMA, F. V.; GEORGAKIS, C. Design of output constraints for model-based non-square controllers using interval operability. Journal of Process Control, Elsevier, v. 18, n. 6, p. 610-620, 2007.

MAYNE, D. Q.; RAWLINGS, J. B.; RAO, C. V.; SCOKAERT, P. O. M. Constrained model predictive control: Stability and optimality. Automatica, Elsevier, n. 36, p. 789-814, 2000.

VADIGEPALLI, R.; GATZKE, E. P.; DOYLE, F. J. Robust control of a multivariable experimental four-tank system. Industrial \& Engineering Chemistry Research, v. 40, n. 8, p. 1916-1927, 2001. 